\title{
Compression of Measured 2D UWB Antenna Transfer Functions
}

\author{
Wouter Dullaert Grzegorz Adamiuk Hendrik Rogier
}

Address the email to:

Wouter Dullaert,

Ghent University, Department of Information Technology

Sint-Pietersnieuwstraat 41, 9000 GENT , Belgium

Phone: +3292643330

Fax: +3292649969

E-mail:wouter.dullaert@intec.ugent.be 


\title{
Compression of Measured 2D UWB Antenna Transfer Functions
}

\author{
Wouter Dullaert Grzegorz Adamiuk Hendrik Rogier
}

\begin{abstract}
We derive rules of thumb for compressing transfer functions of UWB antennas using a phase-mode/Slepian-mode based model. The are applied to measured 2D UWB radiation patterns of bowtie, log-periodic and Vivaldi antennas. A compression ratio of 5.8 with a maximal error of $10 \%$ is achieved.
\end{abstract}

Introduction Stemming from military radar applications, Ultra Wideband (UWB) communications (systems with a relative bandwidth in excess of 0.2 times the center frequency $f_{c}$ or with absolute bandwidth larger than $500 \mathrm{MHz}$ ) are being researched intensively in both academic and industrial environments. In 2002 the FCC licensed UWB communication between $3.1 \mathrm{GHz}$ and $10.6 \mathrm{GHz}$, with a spectral mask of $-41 \mathrm{~dB} / \mathrm{MHz}$, [1], while in 2007 the European Commission allowed UWB communication between $6.0 \mathrm{GHz}$ and $8.5 \mathrm{GHz}$. Because of the large bandwidths, antenna parameters can no longer be considered constant over frequency. Hence, in a UWB system, the frequency dependency of all parameters should be carefully taken into account, resulting in a large increase of data to be manipulated. [2] proposed an efficient model to compress the 3D transfer function of an antenna without loss of accuracy. The main advantage of this model over more traditional rational modeling methods, is that it allows to reduce the noise contribution in the data, provided truncation boundaries are chosen correctly. In this letter, we define rules of thumb for these truncation 
boundaries and apply them to measured 2D UWB radiation patterns of bowtie, log-periodic and Vivaldi antennas.

Compression Ratio We consider the measured vector transfer function of an antenna, which relates the input pulse to the radiated field, as defined in [3]. In this letter elevation angle is held constant at $\theta_{0}$. Since both polarisations can be treated independently we focus on a scalar transfer function $H\left(\theta_{0}, \phi, f\right)$. The model [2] expands the azimuthal dependency into phase modes and the frequency dependency into Slepian modes:

$$
H\left(\theta_{0}, \phi, f\right)=\sum_{m=-M}^{M} \sum_{k=0}^{K} H_{m, k}^{\phi, f}\left(\theta_{0}\right) \psi_{k, c}(f) \exp (j m \phi)
$$

where $\psi_{k, c}(f)$ is the $k$-th order DPSS with bandwidth parameter $c$.

As for the compression ratio of the phase modes, first note that the transfer function is closely related to the radiation pattern:

$$
\frac{\boldsymbol{F}(\theta, \phi, f)}{\sqrt{Z_{0}}}=\frac{\boldsymbol{H}(\theta, \phi, f)}{2 \pi c_{0}} \cdot j \omega \frac{U(f)}{\sqrt{Z_{c}}}
$$

The radiation pattern in the frequency domain is identical to the transfer function, up to a constant factor. From [4], we know that the energy contained in the $m$-th phase mode decreases rapidly when $m>k_{0} r$, with $k_{0}=2 \pi / \lambda$ and $\lambda$ the wavelength. As a rule of thumb we now choose the truncation boundary at $m=\left\lceil k_{0} d\right\rceil+4$, with $d$ the largest dimension of the antenna and $k_{0}$ the wavenumber of the largest frequency under consideration.

To deduct a rule of thumb for the amount of Slepian Modes needed to represent an antenna transfer function we apply some properties of the DPSS derived by Slepian, [5]. Given that the DPSSs are the eigenvectors of the sincmatrix

$$
B(N, c)_{k, l}=\frac{\sin (2 \pi c(k-l))}{\pi(k-l)},
$$


where $0<c<0.5$ is the bandwidth parameter and $N$ the total number of frequency samples, for the corresponding eigenvalues, we find that $1>\lambda_{0}>$ $\lambda_{1}>\lambda_{2}>\lambda_{3}>\ldots \lambda_{n}>0$. These $\lambda_{i}$ are almost 1 for $i \leq 2 c N$ and almost 0 for $i>2 c N$. As these $\lambda_{i}$ also represent the fraction of the spectral energy of the $i$-th order DPSS in $|f|<B W$, with $B W$ the bandwidth on which the DPSS are defined, Parceval's theorem dictates that all $B W$-bandlimited functions are described accurately using only the first $2 c N$ DPSSs. Since there is a small transitional area where the $\lambda_{i}$ cannot be considered close to 1 or 0 , it is advisable to use $K>2 c N$. When $K=\lceil 2 c N\rceil+14$, accuracies over $90 \%$ can be achieved. For large $N$ the influence of this transitional region in the eigenvalues is neglible, but for smaller $N$ it is important to add the extra 14 modes.

Validation To validate these results three different UWB antenna transfer functions, measured at the Universität Karlsruhe [3], were compressed: a bowtie antenna, a log-periodic antenna and a vivaldi antenna. All antennas were measured from $0.4 \mathrm{GHz}$ to $20 \mathrm{GHz}$ at 801 frequency points, using 91 samples in the azimuthal plane. Given that all antennas were measured in the same frequency range, they are expanded into an equal amount of Slepian modes: $801 \times 0.1254 \times 2+14=214$, using $c=0.1254$ as in [2]. The bowtie antenna has dimensions $36 \times 31 \mathrm{~mm}^{2}$, requiring $0.036 \times 20 e 9 / 3 e 8 \times 2 \pi+4=20$ phase modes. In Figure 1 the phase- and Slepian mode coefficients are shown. It is easily verified that the energy at the truncation boundaries is close to zero. For the Vivaldi antenna, measuring $75 \times 78 \mathrm{~mm}^{2}$, we need 37 phase modes. The logperiodic antenna with dimensions $60 \times 50 \mathrm{~mm}^{2}$ is modelled by 30 phase modes. The total number of coefficients needed to describe the frequency response reduces from 72891 to 12626 on average: a reduction by a factor of about 5.8.

The relative errors, between the original transfer function $H\left(\theta_{0}, \phi, f\right)$ and the reconstructed $\hat{H}\left(\theta_{0}, \phi, f\right)$ is defined as $\sum_{\phi}\left|H\left(\theta_{0}, \phi, f\right)-\hat{H}\left(\theta_{0}, \phi, f\right)\right| / \sum_{\phi}\left|H\left(\theta_{0}, \phi, f\right)\right|$ and are shown in Figure 2. We see that all the error curves are very close to 
each other and below $10 \%$ error for almost all frequencies. This can also be verified on Figure 3 , where both the original and reconstructed transfer function, for $\phi=0$, is plotted for all three antennas.

Conclusion Strategies for the accurate prediction of the truncation boundaries of a model for the antenna frequency response using phase and Slepian modes were derived. These rules of thumb were then verified on three measured UWB antennas with a different radiation mechanisms. Using this model the size of the frequency response was compressed by factor of about 5.8 with a relative error below $10 \%$.

\section{References}

[1] New public safety applications and broadband internet access among uses envisioned by FCC authorization of ultra-wideband technology-FCC news release 2002 [online]. http://www.fcc .gov/Bureaus/Engineering_ Technology/News_Releases/2002/nret0203.html.

[2] Wouter Dullaert and Hendrik Rogier. Novel Compact Model for the Radiation Pattern of UWB Antenna using Vector Spherical and Slepian Decomposition. IEEE Transactions on Antennas and Propagation, In press.

[3] W. Wiesbeck, G. Adamiuk, and C. Sturm. Basic Properties and Design Principles of UWB Antennas. Proceedings of the IEEE, 97(2):372-385, Feb. 2009.

[4] Hendrik Rogier and Ernst Bonek. Analytical spherical-mode-based compensation of mutual coupling in uniform circular arrays for direction-ofarrival estimation. Archiv fur Elektronik und Übertragungstechnik (AEÜ)International Journal of Electronics and Communications, 60(3):179-189, March 2006. 
[5] D. Slepian. Prolate spheroidal wave functions, Fourier analysis, and uncertainty. V - The discrete case. AT T Technical Journal, 57:1371-1430, jun 1978.

\section{Affiliations}

Wouter Dullaert and Hendrik Rogier (Ghent Univerity, INTEC, Sint-Pietersnieuwstraat 419000 Ghent, Belgium)

Grzegorz Adamiuk (Karlsruhe Institute of Technology (KIT), Institut fuer Hochfrequenztechnik und Elektronik, Kaiserstr. 12, 76131 Karlsruhe, Germany) 


\section{List of Figures}

1 Model coefficients of the bowtie antenna . . . . . . . . . . 8

2 Relative error ................... 9

3 Antenna transfer function for $\phi=0 \ldots \ldots \ldots$ 


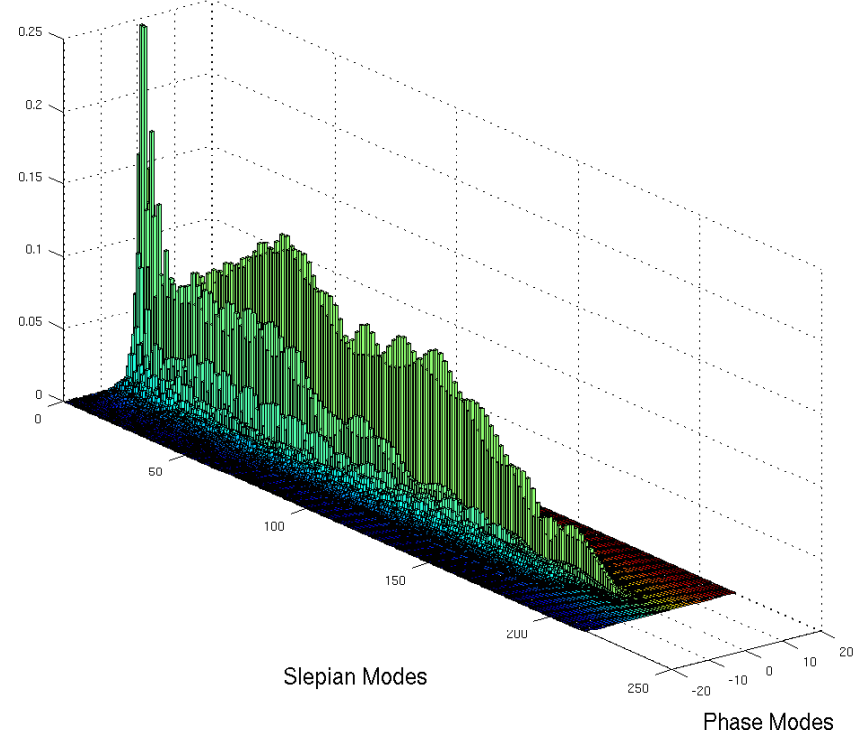

Figure 1: Model coefficients of the bowtie antenna 


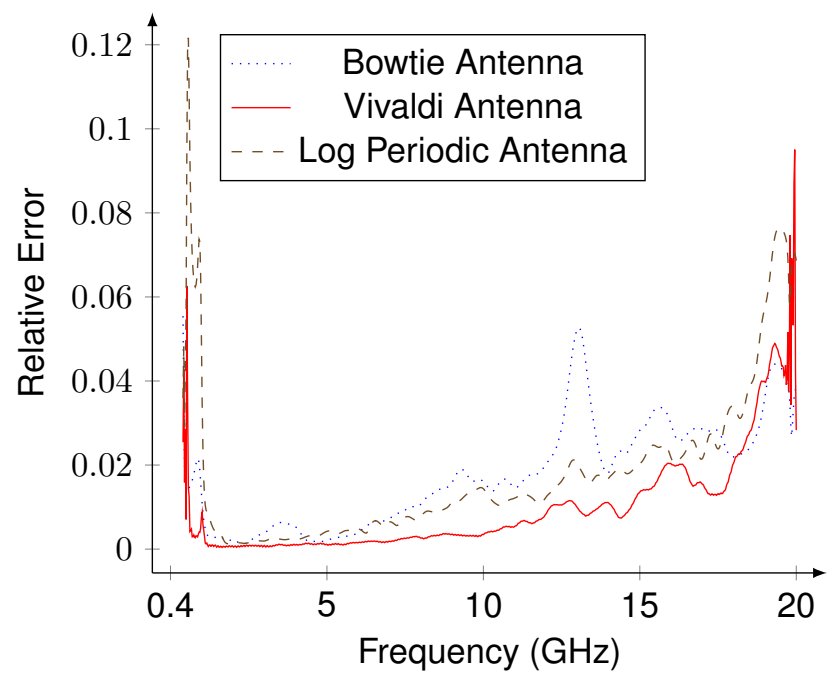

Figure 2: Relative error 


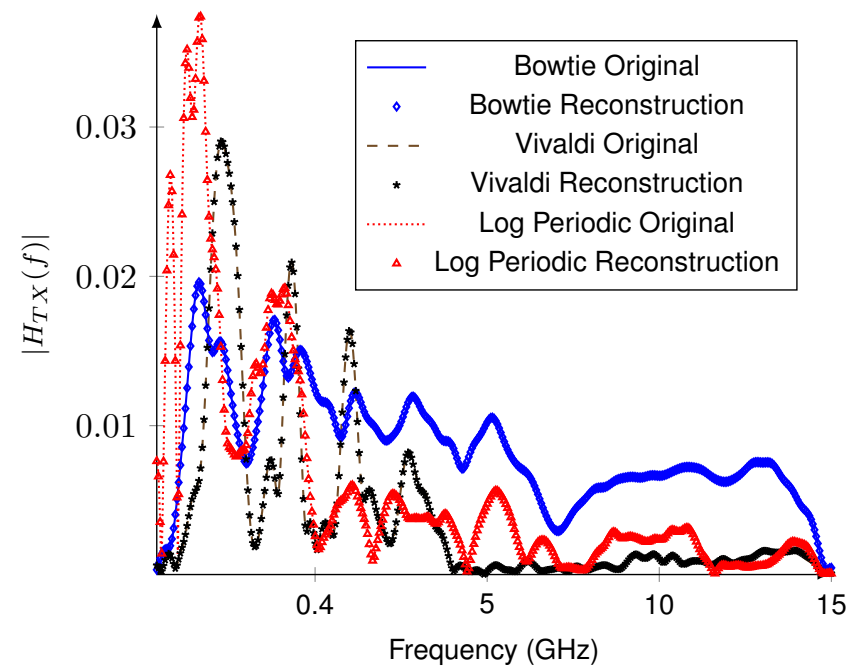

Figure 3: Antenna transfer function for $\phi=0$ 